\title{
ERROR ESTIMATES OF MIXED FINITE ELEMENT APPROXIMATIONS FOR A CLASS OF FOURTH ORDER ELLIPTIC CONTROL PROBLEMS
}

\author{
Tianliang Hou
}

\begin{abstract}
In this paper, we consider the error estimates of the numerical solutions of a class of fourth order linear-quadratic elliptic optimal control problems by using mixed finite element methods. The state and co-state are approximated by the order $k$ Raviart-Thomas mixed finite element spaces and the control variable is approximated by piecewise polynomials of order $k(k \geq 1)$. $L^{2}$ and $L^{\infty}$-error estimates are derived for both the control and the state approximations. These results are seemed to be new in the literature of the mixed finite element methods for fourth order elliptic control problems.
\end{abstract}

\section{Introduction}

There have been many works on finite element methods for the fourth order partial differential equations (PDEs, for short), of course, containing the bi-harmonic equation as well, such as in $[2,9,17,19]$ and so on. The problems described by bi-harmonic equations arise from fluid mechanics and solid mechanics, such as bending of elastic plates. For the fourth order PDEs, the mixed finite elements scheme is naturally introduced, which will reduce the order of PDEs in the mixed system so as to be solved easily. There has been much research about mixed finite element methods for the 4th order PDEs, for example, Ciarlet-Raviart elements, Herrmann-Miyoshi elements, HellanHerrmann-Johnson elements. More details can be found in $[2,9,13,17,19]$ and the references cited therein. Optimal control problems governed by the fourth order PDEs also are encountered in many engineering applications. In [14], Li and Liu introduced a mixed finite element method for the optimal boundary control problem governed by the bi-harmonic equation. Recently, in [3], Cao and Yang derived the a priori error estimates of Ciarlet-Raviart mixed finite element methods for fourth order elliptic control problems.

Received May 23, 2012.

2010 Mathematics Subject Classification. 49J20, 65N30.

Key words and phrases. fourth order elliptic equations, optimal control problems, error estimates, mixed finite element methods. 
In many control problems, the objective functional contains the gradient of the state variables. Thus, the accuracy of the gradient is important in numerical discretization of the coupled state equations. Mixed finite element methods are appropriate for the state equations in such cases since both the scalar variable and its flux variable can be approximated to the same accuracy by using such methods (see, for example, [1]). When the objective functional contains the gradient of the state variable, mixed finite element methods should be used for discretization of the state equation with which both the scalar variable and its flux variable can be approximated in the same accuracy. Recently, in $[5,6,7,8]$, Chen et al. have done some primary works on a priori, superconvergence and a posteriori error estimates for linear elliptic optimal control problems by mixed finite element methods.

In this paper, we will study the $L^{2}$ and $L^{\infty}$-error estimates for a fourth order quadratic elliptic optimal control problem by higher order Raviart-Thomas mixed finite element methods. We consider the following linear-quadratic optimal control problems:

$$
\min _{u \in U_{a d}}\left\{\frac{1}{2}\|\Delta y\|^{2}+\frac{1}{2}\|\nabla y\|^{2}+\frac{1}{2}\left\|y-y_{d}\right\|^{2}+\frac{\nu}{2}\|u\|^{2}\right\}
$$

subject to the state equation

$$
\Delta^{2} y=f+u, \quad x \in \Omega
$$

with the boundary condition

$$
y=\Delta y=0, \quad x \in \partial \Omega,
$$

where $\Omega \subset \mathbb{R}^{2}$ is a bounded domain with Lipschitz continuous boundary. $U_{a d}$ denotes the admissible set of the control variable, defined by

$$
U_{a d}=\left\{u \in L^{\infty}(\Omega): \int_{\Omega} u d x \geq 0\right\} .
$$

Moreover, $f$ and $y_{d}$ are given functions, $\nu$ is a fixed positive number.

The plan of this paper is as follows. In Section 2, we construct the mixed finite element approximation scheme for the optimal control problem (1.1)-(1.3) and give its equivalent optimality conditions. The main results of this paper are stated in Section 3, we derive the $L^{2}$ and $L^{\infty}$-error estimates for both the control and the state approximations. In Section 4, we present a numerical example to demonstrate our theoretical results.

In this paper, we adopt the standard notation $W^{m, p}(\Omega)$ for Sobolev spaces on $\Omega$ with a norm $\|\cdot\|_{m, p}$ given by $\|v\|_{m, p}^{p}=\sum_{|\alpha| \leq m}\left\|D^{\alpha} v\right\|_{L^{p}(\Omega)}^{p}$, a seminorm $|\cdot|_{m, p}$ given by $|v|_{m, p}^{p}=\sum_{|\alpha|=m}\left\|D^{\alpha} v\right\|_{L^{p}(\Omega)}^{p}$. We set $W_{0}^{m, p}(\Omega)=\{v \in$ $\left.W^{m, p}(\Omega):\left.v\right|_{\partial \Omega}=0\right\}$. For $p=2$, we denote $H^{m}(\Omega)=W^{m, 2}(\Omega), H_{0}^{m}(\Omega)=$ $W_{0}^{m, 2}(\Omega)$, and $\|\cdot\|_{m}=\|\cdot\|_{m, 2},\|\cdot\|=\|\cdot\|_{0,2}$. In addition $C$ or $c$ denotes a general positive constant independent of $h$, where $h$ is the spatial mesh-size for the control and state discretization. 


\section{Mixed methods for optimal control problems}

In this section, we shall construct mixed finite element approximation scheme of the control problem (1.1)-(1.3). For sake of simplicity, we assume that the domain $\Omega$ is a convex polygon.

The domain $\Omega$ is said to be $H^{s+2}$-regular if the Dirichlet problem

$$
-\Delta \phi=\psi \quad \text { in } \Omega,\left.\phi\right|_{\partial \Omega}=0
$$

is uniquely solvable for $\psi \in L^{2}(\Omega)$ and if

$$
\|\phi\|_{s+2} \leq C\|\psi\|_{s}
$$

for all $\psi \in H^{s}(\Omega)$.

In the rest of the paper, we assume that the domain $\Omega$ is $H^{k+2}$-regular for $k \geq 1$ and that $f \in H^{k}(\Omega)$ and $y_{d} \in H^{k}(\Omega)$.

Let $\tilde{\boldsymbol{p}}=-\nabla y$ and $\tilde{y}=-\Delta y$. Then the optimal control problem (1.1)-(1.3) is equal to

$$
\min _{u \in U_{a d}}\left\{\frac{1}{2}\|\tilde{y}\|^{2}+\frac{1}{2}\|\tilde{\boldsymbol{p}}\|^{2}+\frac{1}{2}\left\|y-y_{d}\right\|^{2}+\frac{\nu}{2}\|u\|^{2}\right\}
$$

subject to

$$
\begin{aligned}
& \tilde{\boldsymbol{p}}=-\nabla y, \quad x \in \Omega, \\
& \operatorname{div} \tilde{\boldsymbol{p}}=\tilde{y}, \quad x \in \Omega, \\
& \boldsymbol{p}=-\nabla \tilde{y}, \quad x \in \Omega, \\
& \operatorname{div} \boldsymbol{p}=f+u, \quad x \in \Omega, \\
& y=\tilde{y}=0, \quad x \in \partial \Omega .
\end{aligned}
$$

Let

$$
\boldsymbol{V}=H(\operatorname{div} ; \Omega)=\left\{\boldsymbol{v} \in\left(L^{2}(\Omega)\right)^{2}, \operatorname{div} \boldsymbol{v} \in L^{2}(\Omega)\right\}, \quad W=L^{2}(\Omega) .
$$

We recast (2.3)-(2.8) as the following weak form: find $(\boldsymbol{p}, \tilde{y}, \tilde{\boldsymbol{p}}, y, u) \in(\boldsymbol{V} \times$ $W)^{2} \times U_{a d}$ such that

$$
\begin{aligned}
& \min _{u \in U_{a d}}\left\{\frac{1}{2}\|\tilde{y}\|^{2}+\frac{1}{2}\|\tilde{\boldsymbol{p}}\|^{2}+\frac{1}{2}\left\|y-y_{d}\right\|^{2}+\frac{\nu}{2}\|u\|^{2}\right\}, \\
& (\tilde{\boldsymbol{p}}, \boldsymbol{v})-(y, \operatorname{div} \boldsymbol{v})=0, \forall \boldsymbol{v} \in \boldsymbol{V}, \\
& (\operatorname{div} \tilde{\boldsymbol{p}}, w)=(\tilde{y}, w), \forall w \in W, \\
& (\boldsymbol{p}, \boldsymbol{v})-(\tilde{y}, \operatorname{div} \boldsymbol{v})=0, \forall \boldsymbol{v} \in \boldsymbol{V}, \\
& (\operatorname{div} \boldsymbol{p}, w)=(f+u, w), \forall w \in W .
\end{aligned}
$$

It follows from [16] that the above optimal control problem (2.10)-(2.14) has a unique solution $(\boldsymbol{p}, \tilde{y}, \tilde{\boldsymbol{p}}, y, u) \in(\boldsymbol{V} \times W)^{2} \times U_{a d}$, and that $(\boldsymbol{p}, \tilde{y}, \tilde{\boldsymbol{p}}, y, u)$ is the solution of (2.10)-(2.14) if and only if there is a co-state $(\boldsymbol{q}, \tilde{z}, \tilde{\boldsymbol{q}}, z) \in(\boldsymbol{V} \times W)^{2}$ such that $(\boldsymbol{p}, \tilde{y}, \tilde{\boldsymbol{p}}, y, \boldsymbol{q}, \tilde{z}, \tilde{\boldsymbol{q}}, z, u)$ satisfies the following optimality conditions:

$$
(\tilde{\boldsymbol{p}}, \boldsymbol{v})-(y, \operatorname{div} \boldsymbol{v})=0, \forall \boldsymbol{v} \in \boldsymbol{V},
$$




$$
\begin{aligned}
& (\operatorname{div} \tilde{\boldsymbol{p}}, w)=(\tilde{y}, w), \forall w \in W, \\
& (\boldsymbol{p}, \boldsymbol{v})-(\tilde{y}, \operatorname{div} \boldsymbol{v})=0, \forall \boldsymbol{v} \in \boldsymbol{V}, \\
& (\operatorname{div} \boldsymbol{p}, w)=(f+u, w), \forall w \in W, \\
& (\tilde{\boldsymbol{q}}, \boldsymbol{v})-(z, \operatorname{div} \boldsymbol{v})=0, \forall \boldsymbol{v} \in \boldsymbol{V}, \\
& (\operatorname{div} \tilde{\boldsymbol{q}}, w)=(\tilde{z}, w)+(\tilde{y}, w), \forall w \in W, \\
& (\boldsymbol{q}, \boldsymbol{v})-(\tilde{z}, \operatorname{div} \boldsymbol{v})=-(\tilde{\boldsymbol{p}}, \boldsymbol{v}), \forall \boldsymbol{v} \in \boldsymbol{V}, \\
& (\operatorname{div} \boldsymbol{q}, w)=\left(y-y_{d}, w\right), \forall w \in W, \\
& (\nu u+z, \tilde{u}-u) \geq 0, \forall \tilde{u} \in U_{a d},
\end{aligned}
$$

where $(\cdot, \cdot)$ is the inner product of $L^{2}(\Omega)$.

In [22], the expression of the control variable is given as follows:

$$
u=(\max \{0, \bar{z}\}-z) / \nu,
$$

where $\bar{z}=\int_{\Omega} z / \int_{\Omega} 1$ denotes the integral average on $\Omega$ of the function $z$.

Let $\mathcal{T}_{h}$ denote a regular triangulation of the domain $\Omega, h_{T}$ denote the diameter of $T$ and $h=\max h_{T}$. Let $\boldsymbol{V}_{h} \times W_{h} \subset \boldsymbol{V} \times W$ denote the order $k(k \geq 1)$ Raviart-Thomas mixed finite element space with the partition $\mathcal{T}_{h}$ $[10,18]$, namely,

$$
\forall T \in \mathcal{T}_{h}, \quad \boldsymbol{V}(T)=\boldsymbol{P}_{k}(T) \oplus \operatorname{span}\left(x P_{k}(T)\right), \quad W(T)=P_{k}(T),
$$

where $P_{k}(T)$ denotes polynomials of total degree at most $k, \boldsymbol{P}_{k}(T)=\left(P_{k}(T)\right)^{2}$, $x=\left(x_{1}, x_{2}\right)$, which is treated as a vector, and

$$
\begin{aligned}
& V_{h}:=\left\{\boldsymbol{v}_{h} \in \boldsymbol{V}: \forall T \in \mathcal{T}_{h},\left.\boldsymbol{v}_{h}\right|_{T} \in \boldsymbol{V}(T)\right\}, \\
& W_{h}:=\left\{w_{h} \in W: \forall T \in \mathcal{T}_{h},\left.w_{h}\right|_{T} \in W(T)\right\}, \\
& U_{h}:=\left\{\tilde{u}_{h} \in U_{a d}: \forall T \in \mathcal{T}_{h},\left.\tilde{u}_{h}\right|_{T} \in W(T)\right\} .
\end{aligned}
$$

Before the mixed finite element scheme is given, we introduce two operators. Firstly, we define the standard $L^{2}(\Omega)$-projection $[10] P_{h}: W \rightarrow W_{h}$, which satisfies: for any $\phi \in W$

$$
\begin{aligned}
& \left(P_{h} \phi-\phi, w_{h}\right)=0, \forall w_{h} \in W_{h}, \\
& \left\|\phi-P_{h} \phi\right\|_{0, q} \leq C h^{t}\|\phi\|_{t, q}, 0 \leq t \leq k+1, \text { if } \phi \in W^{t, q}(\Omega), \\
& \left\|\phi-P_{h} \phi\right\|_{-r} \leq C h^{r+t}\|\phi\|_{t}, 0 \leq r, t \leq k+1, \text { if } \phi \in H^{t}(\Omega) .
\end{aligned}
$$

Next, recall the Fortin projection (see [1] and [18]) $\Pi_{h}: \boldsymbol{V} \rightarrow \boldsymbol{V}_{h}$, which satisfies: for any $\boldsymbol{q} \in \boldsymbol{V}$

(2.31) $\left(\operatorname{div}\left(\Pi_{h} \boldsymbol{q}-\boldsymbol{q}\right), w_{h}\right)=0, \forall w_{h} \in W_{h}$,

(2.32) $\left\|\boldsymbol{q}-\Pi_{h} \boldsymbol{q}\right\|_{0, q} \leq C h^{t}\|\boldsymbol{q}\|_{t, q}, 1 / q<t \leq k+1$, if $\boldsymbol{q} \in\left(W^{t, q}(\Omega)\right)^{2}$,

(2.33) $\left\|\operatorname{div}\left(\boldsymbol{q}-\Pi_{h} \boldsymbol{q}\right)\right\|_{0, q} \leq C h^{t}\|\operatorname{div} \boldsymbol{q}\|_{t, q}, 0 \leq t \leq k+1$, if $\operatorname{div} \boldsymbol{q} \in W^{t, q}(\Omega)$.

We have the commuting diagram property

$$
\operatorname{div} \circ \Pi_{h}=P_{h} \circ \operatorname{div}: \boldsymbol{V} \rightarrow W_{h} \quad \text { and } \quad \operatorname{div}\left(I-\Pi_{h}\right) \boldsymbol{V} \perp W_{h},
$$


where and after, $I$ denotes identity operator.

Then the mixed finite element discretization of (2.10)-(2.14) is as follows: find $\left(\boldsymbol{p}_{h}, \tilde{y}_{h}, \tilde{\boldsymbol{p}}_{h}, y_{h}, u_{h}\right) \in\left(\boldsymbol{V}_{h} \times W_{h}\right)^{2} \times U_{h}$ such that

$$
\begin{aligned}
& \min _{u_{h} \in U_{h}}\left\{\frac{1}{2}\left\|\tilde{y}_{h}\right\|^{2}+\frac{1}{2}\left\|\tilde{\boldsymbol{p}}_{h}\right\|^{2}+\frac{1}{2}\left\|y_{h}-y_{d}\right\|^{2}+\frac{\nu}{2}\left\|u_{h}\right\|^{2}\right\}, \\
& \left(\tilde{\boldsymbol{p}}_{h}, \boldsymbol{v}_{h}\right)-\left(y_{h}, \operatorname{div} \boldsymbol{v}_{h}\right)=0, \forall \boldsymbol{v}_{h} \in \boldsymbol{V}_{h}, \\
& \left(\operatorname{div} \tilde{\boldsymbol{p}}_{h}, w_{h}\right)=\left(\tilde{y}_{h}, w_{h}\right), \forall w_{h} \in W_{h}, \\
& \left(\boldsymbol{p}_{h}, \boldsymbol{v}_{h}\right)-\left(\tilde{y}_{h}, \operatorname{div} \boldsymbol{v}_{h}\right)=0, \forall \boldsymbol{v}_{h} \in \boldsymbol{V}_{h}, \\
& \left(\operatorname{div} \boldsymbol{p}_{h}, w_{h}\right)=\left(f+u_{h}, w_{h}\right), \forall w_{h} \in W_{h} .
\end{aligned}
$$

The optimal control problem (2.35)-(2.39) has a unique solution $\left(\boldsymbol{p}_{h}, \tilde{y}_{h}, \tilde{\boldsymbol{p}}_{h}\right.$, $\left.y_{h}, u_{h}\right) \in\left(\boldsymbol{V}_{h} \times W_{h}\right)^{2} \times U_{h}$, and that $\left(\boldsymbol{p}_{h}, \tilde{y}_{h}, \tilde{\boldsymbol{p}}_{h}, y_{h}, u_{h}\right)$ is the solution of (2.35)(2.39) if and only if there is a co-state $\left(\boldsymbol{q}_{h}, \tilde{z}_{h}, \tilde{\boldsymbol{q}}_{h}, z_{h}\right) \in\left(\boldsymbol{V}_{h} \times W_{h}\right)^{2}$ such that $\left(\boldsymbol{p}_{h}, \tilde{y}_{h}, \tilde{\boldsymbol{p}}_{h}, y_{h}, \boldsymbol{q}_{h}, \tilde{z}_{h}, \tilde{\boldsymbol{q}}_{h}, z_{h}, u_{h}\right)$ satisfies the following optimality conditions:

$$
\begin{aligned}
& \left(\tilde{\boldsymbol{p}}_{h}, \boldsymbol{v}_{h}\right)-\left(y_{h}, \operatorname{div} \boldsymbol{v}_{h}\right)=0, \forall \boldsymbol{v}_{h} \in \boldsymbol{V}_{h}, \\
& \left(\operatorname{div} \tilde{\boldsymbol{p}}_{h}, w_{h}\right)=\left(\tilde{y}_{h}, w_{h}\right), \forall w_{h} \in W_{h}, \\
& \left(\boldsymbol{p}_{h}, \boldsymbol{v}_{h}\right)-\left(\tilde{y}_{h}, \operatorname{div} \boldsymbol{v}_{h}\right)=0, \forall \boldsymbol{v}_{h} \in \boldsymbol{V}_{h}, \\
& \left(\operatorname{div} \boldsymbol{p}_{h}, w_{h}\right)=\left(f+u_{h}, w_{h}\right), \forall w_{h} \in W_{h}, \\
& \left(\tilde{\boldsymbol{q}}_{h}, \boldsymbol{v}_{h}\right)-\left(z_{h}, \operatorname{div} \boldsymbol{v}_{h}\right)=0, \forall \boldsymbol{v}_{h} \in \boldsymbol{V}_{h}, \\
& \left(\operatorname{div} \tilde{\boldsymbol{q}}_{h}, w_{h}\right)=\left(\tilde{z}_{h}, w_{h}\right)+\left(\tilde{y}_{h}, w_{h}\right), \forall w_{h} \in W_{h}, \\
& \left(\boldsymbol{q}_{h}, \boldsymbol{v}_{h}\right)-\left(\tilde{z}_{h}, \operatorname{div} \boldsymbol{v}_{h}\right)=-\left(\tilde{\boldsymbol{p}}_{h}, \boldsymbol{v}_{h}\right), \forall \boldsymbol{v}_{h} \in \boldsymbol{V}_{h}, \\
& \left(\operatorname{div} \boldsymbol{q}_{h}, w_{h}\right)=\left(y_{h}-y_{d}, w_{h}\right), \forall w_{h} \in W_{h}, \\
& \left(\nu u_{h}+z_{h}, \tilde{u}_{h}-u_{h}\right) \geq 0, \forall \tilde{u}_{h} \in U_{h} .
\end{aligned}
$$

In the rest of the paper, we shall use some intermediate variables. We define the discrete state solution $\left(\boldsymbol{p}_{h}(\tilde{u}), \tilde{y}_{h}(\tilde{u}), \tilde{\boldsymbol{p}}_{h}(\tilde{u}), y_{h}(\tilde{u}), \boldsymbol{q}_{h}(\tilde{u}), \tilde{z}_{h}(\tilde{u}), \tilde{\boldsymbol{q}}_{h}(\tilde{u})\right.$, $\left.z_{h}(\tilde{u})\right) \in\left(\boldsymbol{V}_{h} \times W_{h}\right)^{4}$ associated with $\tilde{u}$ that satisfies

$$
\begin{aligned}
& \left(\tilde{\boldsymbol{p}}_{h}(\tilde{u}), \boldsymbol{v}_{h}\right)-\left(y_{h}(\tilde{u}), \operatorname{div} \boldsymbol{v}_{h}\right)=0, \forall \boldsymbol{v}_{h} \in \boldsymbol{V}_{h}, \\
& \left(\operatorname{div} \tilde{\boldsymbol{p}}_{h}(\tilde{u}), w_{h}\right)=\left(\tilde{y}_{h}(\tilde{u}), w_{h}\right), \forall w_{h} \in W_{h}, \\
& \left(\boldsymbol{p}_{h}(\tilde{u}), \boldsymbol{v}_{h}\right)-\left(\tilde{y}_{h}(\tilde{u}), \operatorname{div} \boldsymbol{v}_{h}\right)=0, \forall \boldsymbol{v}_{h} \in \boldsymbol{V}_{h}, \\
& \left(\operatorname{div} \boldsymbol{p}_{h}(\tilde{u}), w_{h}\right)=\left(f+\tilde{u}, w_{h}\right), \forall w_{h} \in W_{h}, \\
& \left(\tilde{\boldsymbol{q}}_{h}(\tilde{u}), \boldsymbol{v}_{h}\right)-\left(z_{h}(\tilde{u}), \operatorname{div} \boldsymbol{v}_{h}\right)=0, \forall \boldsymbol{v}_{h} \in \boldsymbol{V}_{h}, \\
& \left(\operatorname{div} \tilde{\boldsymbol{q}}_{h}(\tilde{u}), w_{h}\right)=\left(\tilde{z}_{h}(\tilde{u}), w_{h}\right)+\left(\tilde{y}_{h}(\tilde{u}), w_{h}\right), \forall w_{h} \in W_{h}, \\
& \left(\boldsymbol{q}_{h}(\tilde{u}), \boldsymbol{v}_{h}\right)-\left(\tilde{z}_{h}(\tilde{u}), \operatorname{div} \boldsymbol{v}_{h}\right)=-\left(\tilde{\boldsymbol{p}}_{h}(\tilde{u}), \boldsymbol{v}_{h}\right), \forall \boldsymbol{v}_{h} \in \boldsymbol{V}_{h}, \\
& \left(\operatorname{div} \boldsymbol{q}_{h}(\tilde{u}), w_{h}\right)=\left(y_{h}(\tilde{u})-y_{d}, w_{h}\right), \forall w_{h} \in W_{h} .
\end{aligned}
$$


Thus, as we defined, the exact solution and its approximation can be written in the following way:

$$
\begin{aligned}
& (\boldsymbol{p}, \tilde{y}, \tilde{\boldsymbol{p}}, y, \boldsymbol{q}, \tilde{z}, \tilde{\boldsymbol{q}}, z)=(\boldsymbol{p}(u), \tilde{y}(u), \tilde{\boldsymbol{p}}(u), y(u), \boldsymbol{q}(u), \tilde{z}(u), \tilde{\boldsymbol{q}}(u), z(u)), \\
& \left(\boldsymbol{p}_{h}, \tilde{y}_{h}, \tilde{\boldsymbol{p}}_{h}, y_{h}, \boldsymbol{q}_{h}, \tilde{z}_{h}, \tilde{\boldsymbol{q}}_{h}, z_{h}\right)=\left(\boldsymbol{p}_{h}\left(u_{h}\right), \tilde{y}_{h}\left(u_{h}\right), \tilde{\boldsymbol{p}}_{h}\left(u_{h}\right), y_{h}\left(u_{h}\right), \boldsymbol{q}_{h}\left(u_{h}\right), \tilde{z}_{h}\left(u_{h}\right)\right. \text {, } \\
& \left.\tilde{\boldsymbol{q}}_{h}\left(u_{h}\right), z_{h}\left(u_{h}\right)\right) \text {. }
\end{aligned}
$$

\section{3. $L^{2}$ and $L^{\infty}$-error estimates}

In this section, we will give the $L^{2}$ and $L^{\infty}$-error estimates both for the control and the state approximation.

Firstly, we easily derive the following convergence results for the intermediate solutions.

Lemma 3.1. Let $\left(\boldsymbol{p}_{h}(u), \tilde{y}_{h}(u), \tilde{\boldsymbol{p}}_{h}(u), y_{h}(u), \boldsymbol{q}_{h}(u), \tilde{z}_{h}(u), \tilde{\boldsymbol{q}}_{h}(u), z_{h}(u)\right) \in\left(\boldsymbol{V}_{h}\right.$ $\left.\times W_{h}\right)^{4}$ be the solution of (2.49)-(2.56) with $\tilde{u}=u$ and $(\boldsymbol{p}, \tilde{y}, \tilde{\boldsymbol{p}}, y, \boldsymbol{q}, \tilde{z}, \tilde{\boldsymbol{q}}, z) \in$ $(\boldsymbol{V} \times W)^{4}$ be the solution of (2.15)-(2.23), respectively. Assume that $y, \tilde{y}, z, \tilde{z} \in$ $H^{k+1}(\Omega), \boldsymbol{p}, \tilde{\boldsymbol{p}}, \boldsymbol{q}, \tilde{\boldsymbol{q}} \in\left(H^{k+1}(\Omega)\right)^{2}$ and divp, div $\tilde{\boldsymbol{p}}, \operatorname{div} \boldsymbol{q}$, div $\tilde{\boldsymbol{q}} \in H^{k+1}(\Omega)$. Then we have

$$
\begin{aligned}
& \left\|y-y_{h}(u)\right\|+\left\|\boldsymbol{p}-\boldsymbol{p}_{h}(u)\right\|_{d i v} \leq C h^{k+1}, \\
& \left\|z-z_{h}(u)\right\|+\left\|\boldsymbol{q}-\boldsymbol{q}_{h}(u)\right\|_{d i v} \leq C h^{k+1}, \\
& \left\|\tilde{y}-\tilde{y}_{h}(u)\right\|+\left\|\tilde{\boldsymbol{p}}-\tilde{\boldsymbol{p}}_{h}(u)\right\|_{d i v} \leq C h^{k+1}, \\
& \left\|\tilde{z}-\tilde{z}_{h}(u)\right\|+\left\|\tilde{\boldsymbol{q}}-\tilde{\boldsymbol{q}}_{h}(u)\right\|_{d i v} \leq C h^{k+1} .
\end{aligned}
$$

Proof. From Equations (2.49)-(2.56) and (2.15)-(2.23), we obtain the following error equations

(3.5) $\left(\tilde{\boldsymbol{p}}-\tilde{\boldsymbol{p}}_{h}(u), \boldsymbol{v}_{h}\right)-\left(y-y_{h}(u), \operatorname{div} \boldsymbol{v}_{h}\right)=0, \forall \boldsymbol{v}_{h} \in \boldsymbol{V}_{h}$,

(3.6) $\quad\left(\operatorname{div}\left(\tilde{\boldsymbol{p}}-\tilde{\boldsymbol{p}}_{h}(u)\right), w_{h}\right)=\left(\tilde{y}-\tilde{y}_{h}(u), w_{h}\right), \forall w_{h} \in W_{h}$,

(3.7) $\left(\boldsymbol{p}-\boldsymbol{p}_{h}(u), \boldsymbol{v}_{h}\right)-\left(\tilde{y}-\tilde{y}_{h}(u), \operatorname{div} \boldsymbol{v}_{h}\right)=0, \forall \boldsymbol{v}_{h} \in \boldsymbol{V}_{h}$,

(3.8) $\left(\operatorname{div}\left(\boldsymbol{p}-\boldsymbol{p}_{h}(u)\right), w_{h}\right)=0, \forall w_{h} \in W_{h}$,

(3.9) $\left(\tilde{\boldsymbol{q}}-\tilde{\boldsymbol{q}}_{h}(u), \boldsymbol{v}_{h}\right)-\left(z-z_{h}(u), \operatorname{div} \boldsymbol{v}_{h}\right)=0, \forall \boldsymbol{v}_{h} \in \boldsymbol{V}_{h}$,

$(3.10)\left(\operatorname{div}\left(\tilde{\boldsymbol{q}}-\tilde{\boldsymbol{q}}_{h}(u)\right), w_{h}\right)=\left(\tilde{z}-\tilde{z}_{h}(u), w_{h}\right)+\left(\tilde{y}-\tilde{y}_{h}(u), w_{h}\right), \forall w_{h} \in W_{h}$,

$(3.11)\left(\boldsymbol{q}-\boldsymbol{q}_{h}(u), \boldsymbol{v}_{h}\right)-\left(\tilde{z}-\tilde{z}_{h}(u), \operatorname{div} \boldsymbol{v}_{h}\right)=-\left(\tilde{\boldsymbol{p}}-\tilde{\boldsymbol{p}}_{h}(u), \boldsymbol{v}_{h}\right), \forall \boldsymbol{v}_{h} \in \boldsymbol{V}_{h}$,

(3.12) $\left(\operatorname{div}\left(\boldsymbol{q}-\boldsymbol{q}_{h}(u)\right), w_{h}\right)=\left(y-y_{h}(u), w_{h}\right), \forall w_{h} \in W_{h}$.

It follows from the standard stability estimate, (2.29) and (2.32) that

$$
\begin{gathered}
\left\|\tilde{y}-\tilde{y}_{h}(u)\right\|+\left\|\boldsymbol{p}-\boldsymbol{p}_{h}(u)\right\|_{\operatorname{div}} \leq C h^{k+1}, \\
\left\|y-y_{h}(u)\right\|+\left\|\tilde{\boldsymbol{p}}-\tilde{\boldsymbol{p}}_{h}(u)\right\|_{\operatorname{div}} \leq C h^{k+1}+C\left\|\tilde{y}-\tilde{y}_{h}(u)\right\|,
\end{gathered}
$$

$$
\left\|\tilde{z}-\tilde{z}_{h}(u)\right\|+\left\|\boldsymbol{q}-\boldsymbol{q}_{h}(u)\right\|_{\text {div }} \leq C h^{k+1}+C\left(\left\|y-y_{h}(u)\right\|+\left\|\tilde{\boldsymbol{p}}-\tilde{\boldsymbol{p}}_{h}(u)\right\|\right),
$$


ERROR ESTIMATES FOR FOURTH ORDER ELLIPTIC CONTROL PROBLEMS 1133

$$
\left\|z-z_{h}(u)\right\|+\left\|\tilde{\boldsymbol{q}}-\tilde{\boldsymbol{q}}_{h}(u)\right\|_{\operatorname{div}} \leq C h^{k+1}+C\left(\left\|\tilde{z}-\tilde{z}_{h}(u)\right\|+\left\|\tilde{y}-\tilde{y}_{h}(u)\right\|\right) .
$$

Thus, (3.1)-(3.4) can be proved from (3.13)-(3.16).

Then, we will give the following superconvergence results for the intermediate solutions which are very important for our following work.

Lemma 3.2. Let $\left(\boldsymbol{p}_{h}(u), \tilde{y}_{h}(u), \tilde{\boldsymbol{p}}_{h}(u), y_{h}(u), \boldsymbol{q}_{h}(u), \tilde{z}_{h}(u), \tilde{\boldsymbol{q}}_{h}(u), z_{h}(u)\right) \in\left(\boldsymbol{V}_{h}\right.$ $\left.\times W_{h}\right)^{4}$ be the solution of (2.49)-(2.56) with $\tilde{u}=u$ and $(\boldsymbol{p}, \tilde{y}, \tilde{\boldsymbol{p}}, y, \boldsymbol{q}, \tilde{z}, \tilde{\boldsymbol{q}}, z) \in$ $(\boldsymbol{V} \times W)^{4}$ be the solution of (2.15)-(2.23), respectively. Assume that $y, \tilde{y}, z, \tilde{z} \in$ $H^{k+1}(\Omega), \boldsymbol{p}, \tilde{\boldsymbol{p}}, \boldsymbol{q}, \tilde{\boldsymbol{q}} \in\left(H^{k+1}(\Omega)\right)^{2}$ and divp, div $\tilde{\boldsymbol{p}}$, div $\boldsymbol{q}$, div $\tilde{\boldsymbol{q}} \in H^{k}(\Omega)$. Then we have

$$
\begin{aligned}
&\left\|P_{h} y-y_{h}(u)\right\|+\left\|P_{h} z-z_{h}(u)\right\| \leq C h^{k+2}, \\
&\left\|P_{h} \tilde{y}-\tilde{y}_{h}(u)\right\|+\left\|P_{h} \tilde{z}-\tilde{z}_{h}(u)\right\| \leq C h^{k+2} .
\end{aligned}
$$

Proof. Using (2.28), we can rewrite (3.5)-(3.12) as

$$
\left(\tilde{\boldsymbol{p}}-\tilde{\boldsymbol{p}}_{h}(u), \boldsymbol{v}_{h}\right)-\left(P_{h} y-y_{h}(u), \operatorname{div} \boldsymbol{v}_{h}\right)=0, \forall \boldsymbol{v}_{h} \in \boldsymbol{V}_{h},
$$

$$
\left(\operatorname{div}\left(\tilde{\boldsymbol{p}}-\tilde{\boldsymbol{p}}_{h}(u)\right), w_{h}\right)=\left(P_{h} \tilde{y}-\tilde{y}_{h}(u), w_{h}\right), \forall w_{h} \in W_{h},
$$

$$
\left(\boldsymbol{p}-\boldsymbol{p}_{h}(u), \boldsymbol{v}_{h}\right)-\left(P_{h} \tilde{y}-\tilde{y}_{h}(u), \operatorname{div} \boldsymbol{v}_{h}\right)=0, \forall \boldsymbol{v}_{h} \in \boldsymbol{V}_{h},
$$$$
\left(\operatorname{div}\left(\boldsymbol{p}-\boldsymbol{p}_{h}(u)\right), w_{h}\right)=0, \forall w_{h} \in W_{h},
$$

$$
\left(\tilde{\boldsymbol{q}}-\tilde{\boldsymbol{q}}_{h}(u), \boldsymbol{v}_{h}\right)-\left(P_{h} z-z_{h}(u), \operatorname{div} \boldsymbol{v}_{h}\right)=0, \forall \boldsymbol{v}_{h} \in \boldsymbol{V}_{h},
$$

$\left(\operatorname{div}\left(\tilde{\boldsymbol{q}}-\tilde{\boldsymbol{q}}_{h}(u)\right), w_{h}\right)=\left(P_{h} \tilde{z}-\tilde{z}_{h}(u), w_{h}\right)+\left(P_{h} \tilde{y}-\tilde{y}_{h}(u), w_{h}\right), \forall w_{h} \in W_{h}$,

$$
\left(\boldsymbol{q}-\boldsymbol{q}_{h}(u), \boldsymbol{v}_{h}\right)-\left(P_{h} \tilde{z}-\tilde{z}_{h}(u), \operatorname{div} \boldsymbol{v}_{h}\right)=-\left(\tilde{\boldsymbol{p}}-\tilde{\boldsymbol{p}}_{h}(u), \boldsymbol{v}_{h}\right), \forall \boldsymbol{v}_{h} \in \boldsymbol{V}_{h},
$$

$$
\left(\operatorname{div}\left(\boldsymbol{q}-\boldsymbol{q}_{h}(u)\right), w_{h}\right)=\left(P_{h} y-y_{h}(u), w_{h}\right), \forall w_{h} \in W_{h} .
$$

For sake of simplicity, we now denote

$$
e=P_{h} \tilde{z}-\tilde{z}_{h}(u)
$$

Since

$$
\|e\|=\sup _{\psi \in L^{2}(\Omega), \psi \neq 0} \frac{(e, \psi)}{\|\psi\|},
$$

we then need to bound $(e, \psi)$ for $\psi \in L^{2}(\Omega)$. Let $\phi \in H^{2}(\Omega) \cap H_{0}^{1}(\Omega)$ be the solution of (2.1). We can see from (2.31) and (3.25)

$$
(e, \psi)=(e,-\operatorname{div}(\nabla \phi))=-\left(e, \operatorname{div}\left(\Pi_{h}(\nabla \phi)\right)\right)
$$

$$
=-\left(\boldsymbol{q}-\boldsymbol{q}_{h}(u), \Pi_{h}(\nabla \phi)\right)-\left(\tilde{\boldsymbol{p}}-\tilde{\boldsymbol{p}}_{h}(u), \Pi_{h}(\nabla \phi)\right) .
$$


Note that

$$
\left(\operatorname{div}\left(\boldsymbol{q}-\boldsymbol{q}_{h}(u)\right), \phi\right)+\left(\boldsymbol{q}-\boldsymbol{q}_{h}(u), \nabla \phi\right)=0 .
$$

Thus, from (2.29), (2.32), (2.33), (3.19), (3.26), (3.29), (3.30) and Lemma 3.1, we derive

$$
\begin{aligned}
(e, \psi)= & \left(\boldsymbol{q}-\boldsymbol{q}_{h}(u), \nabla \phi-\Pi_{h}(\nabla \phi)\right) \\
& +\left(\operatorname{div}\left(\boldsymbol{q}-\boldsymbol{q}_{h}(u)\right), \phi-P_{h} \phi\right)+\left(P_{h} y-y_{h}(u), P_{h} \phi\right) \\
& -\left(P_{h} y-y_{h}(u), \operatorname{div}\left(\Pi_{h}(\nabla \phi)\right)\right) \\
\leq & C\left(h^{k+2}+\left\|P_{h} y-y_{h}(u)\right\|\right)\|\phi\|_{2} .
\end{aligned}
$$

Similarly, using the duality argument, we can find that

$$
\begin{aligned}
& \left\|P_{h} \tilde{y}-\tilde{y}_{h}(u)\right\| \leq C h^{k+2}, \\
& \left\|P_{h} y-y_{h}(u)\right\| \leq C h^{k+2}+C\left\|P_{h} \tilde{y}-\tilde{y}_{h}(u)\right\|, \\
& \left\|P_{h} z-z_{h}(u)\right\| \leq C h^{k+2}+C\left(\left\|P_{h} \tilde{y}-\tilde{y}_{h}(u)\right\|+\left\|P_{h} \tilde{z}-\tilde{z}_{h}(u)\right\|\right) .
\end{aligned}
$$

Thus, by (3.28) and (3.31)-(3.34), we complete the proof.

Lemma 3.3. Let $z_{h}\left(P_{h} u\right)$ and $z_{h}$ be the solutions of (2.49)-(2.56) with $\tilde{u}=P_{h} u$ and $\tilde{u}=u_{h}$, respectively, then we have

$$
\left(z_{h}-z_{h}\left(P_{h} u\right), P_{h} u-u_{h}\right) \leq 0 .
$$

Proof. Choose $\tilde{u}=P_{h} u$ and $\tilde{u}=u_{h}$ in (2.49)-(2.56), respectively, we obtain the following error equations:

$$
\begin{aligned}
& \left(\tilde{\boldsymbol{p}}_{h}\left(P_{h} u\right)-\tilde{\boldsymbol{p}}_{h}, \boldsymbol{v}_{h}\right)-\left(y_{h}\left(P_{h} u\right)-y_{h}, \operatorname{div} \boldsymbol{v}_{h}\right)=0, \boldsymbol{v}_{h} \in \boldsymbol{V}_{h}, \\
& \left(\operatorname{div}\left(\tilde{\boldsymbol{p}}_{h}\left(P_{h} u\right)-\tilde{\boldsymbol{p}}_{h}\right), w_{h}\right)=\left(\tilde{y}_{h}\left(P_{h} u\right)-\tilde{y}_{h}, w_{h}\right), w_{h} \in W_{h}, \\
& \left(\boldsymbol{p}_{h}\left(P_{h} u\right)-\boldsymbol{p}_{h}, \boldsymbol{v}_{h}\right)-\left(\tilde{y}_{h}\left(P_{h} u\right)-\tilde{y}_{h}, \operatorname{div} \boldsymbol{v}_{h}\right)=0, \boldsymbol{v}_{h} \in \boldsymbol{V}_{h}, \\
& \left(\operatorname{div}\left(\boldsymbol{p}_{h}\left(P_{h} u\right)-\boldsymbol{p}_{h}\right), w_{h}\right)=\left(P_{h} u-u_{h}, w_{h}\right), w_{h} \in W_{h}, \\
& \left(\tilde{\boldsymbol{q}}_{h}\left(P_{h} u\right)-\tilde{\boldsymbol{q}}_{h}, \boldsymbol{v}_{h}\right)-\left(z_{h}\left(P_{h} u\right)-z_{h}, \operatorname{div} \boldsymbol{v}_{h}\right)=0, \boldsymbol{v}_{h} \in \boldsymbol{V}_{h},
\end{aligned}
$$

$\left(\operatorname{div}\left(\tilde{\boldsymbol{q}}_{h}\left(P_{h} u\right)-\tilde{\boldsymbol{q}}_{h}\right), w_{h}\right)=\left(\tilde{z}_{h}\left(P_{h} u\right)-\tilde{z}_{h}, w_{h}\right)+\left(\tilde{y}_{h}\left(P_{h} u\right)-\tilde{y}_{h}, w_{h}\right), w_{h} \in W_{h}$,

$$
\left(\boldsymbol{q}_{h}\left(P_{h} u\right)-\boldsymbol{q}_{h}, \boldsymbol{v}_{h}\right)-\left(\tilde{z}_{h}\left(P_{h} u\right)-\tilde{z}_{h}, \operatorname{div} \boldsymbol{v}_{h}\right)=-\left(\tilde{\boldsymbol{p}}_{h}\left(P_{h} u\right)-\tilde{\boldsymbol{p}}_{h}, \boldsymbol{v}_{h}\right), \boldsymbol{v}_{h} \in \boldsymbol{V}_{h},
$$

$$
\left(\operatorname{div}\left(\boldsymbol{q}_{h}\left(P_{h} u\right)-\boldsymbol{q}_{h}\right), w_{h}\right)=\left(y_{h}\left(P_{h} u\right)-y_{h}, w_{h}\right), w_{h} \in W_{h} .
$$

For the above equations, we choose $w_{h}=\tilde{z}_{h}-\tilde{z}_{h}\left(P_{h} u\right)$ in $(3.37), \boldsymbol{v}_{h}=\tilde{\boldsymbol{q}}_{h}-$ $\tilde{\boldsymbol{q}}_{h}\left(P_{h} u\right)$ in (3.36), $w_{h}=z_{h}-z_{h}\left(P_{h} u\right)$ in (3.39), $\boldsymbol{v}_{h}=\boldsymbol{q}_{h}-\boldsymbol{q}_{h}\left(P_{h} u\right)$ in (3.38), 
ERROR ESTIMATES FOR FOURTH ORDER ELLIPTIC CONTROL PROBLEMS 1135 $w_{h}=\tilde{y}_{h}-\tilde{y}_{h}\left(P_{h} u\right)$ in $(3.41), \boldsymbol{v}_{h}=\boldsymbol{p}_{h}-\boldsymbol{p}_{h}\left(P_{h} u\right)$ in $(3.40), w_{h}=y_{h}-y_{h}\left(P_{h} u\right)$ in (3.43) and $\boldsymbol{v}_{h}=\tilde{\boldsymbol{p}}_{h}-\tilde{\boldsymbol{p}}_{h}\left(P_{h} u\right)$ in (3.42), then we can deduce that

$$
\begin{aligned}
& \left(z_{h}-z_{h}\left(P_{h} u\right), P_{h} u-u_{h}\right) \\
= & -\left\|\tilde{\boldsymbol{p}}_{h}-\tilde{\boldsymbol{p}}_{h}\left(P_{h} u\right)\right\|^{2}-\left\|y_{h}-y_{h}\left(P_{h} u\right)\right\|^{2}-\left\|\tilde{y}_{h}-\tilde{y}_{h}\left(P_{h} u\right)\right\|^{2} \leq 0,
\end{aligned}
$$

which implies (3.35).

Lemma 3.4. Let $\left(\boldsymbol{p}_{h}\left(P_{h} u\right), \tilde{y}_{h}\left(P_{h} u\right), \tilde{\boldsymbol{p}}_{h}\left(P_{h} u\right), y_{h}\left(P_{h} u\right), \boldsymbol{q}_{h}\left(P_{h} u\right), \tilde{z}_{h}\left(P_{h} u\right)\right.$, $\left.\tilde{\boldsymbol{q}}_{h}\left(P_{h} u\right), z_{h}\left(P_{h} u\right)\right)$ and $\left(\boldsymbol{p}_{h}(u), \tilde{y}_{h}(u), \tilde{\boldsymbol{p}}_{h}(u), y_{h}(u), \boldsymbol{q}_{h}(u), \tilde{z}_{h}(u), \tilde{\boldsymbol{q}}_{h}(u), z_{h}(u)\right)$ be the solutions of (2.49)-(2.56) with $\tilde{u}=P_{h} u$ and $\tilde{u}=u$, respectively. Then we have

$$
\begin{aligned}
& \left\|y_{h}(u)-y_{h}\left(P_{h} u\right)\right\|+\left\|\boldsymbol{p}_{h}(u)-\boldsymbol{p}_{h}\left(P_{h} u\right)\right\|=0, \\
& \left\|z_{h}(u)-z_{h}\left(P_{h} u\right)\right\|+\left\|\boldsymbol{q}_{h}(u)-\boldsymbol{q}_{h}\left(P_{h} u\right)\right\|=0, \\
& \left\|\tilde{y}_{h}(u)-\tilde{y}_{h}\left(P_{h} u\right)\right\|+\left\|\tilde{\boldsymbol{p}}_{h}(u)-\tilde{\boldsymbol{p}}_{h}\left(P_{h} u\right)\right\|=0, \\
& \left\|\tilde{z}_{h}(u)-\tilde{z}_{h}\left(P_{h} u\right)\right\|+\left\|\tilde{\boldsymbol{q}}_{h}(u)-\tilde{\boldsymbol{q}}_{h}\left(P_{h} u\right)\right\|=0 .
\end{aligned}
$$

Proof. First, we choose $\tilde{u}=P_{h} u$ and $\tilde{u}=u$ in (2.49)-(2.56) respectively, then we obtain the following error equations

$$
\begin{gathered}
\left(\tilde{\boldsymbol{p}}_{h}\left(P_{h} u\right)-\tilde{\boldsymbol{p}}_{h}(u), \boldsymbol{v}_{h}\right)-\left(y_{h}\left(P_{h} u\right)-y_{h}(u), \operatorname{div} \boldsymbol{v}_{h}\right)=0, \boldsymbol{v}_{h} \in \boldsymbol{V}_{h}, \\
\left(\operatorname{div}\left(\tilde{\boldsymbol{p}}_{h}\left(P_{h} u\right)-\tilde{\boldsymbol{p}}_{h}(u)\right), w_{h}\right)=\left(\tilde{y}_{h}\left(P_{h} u\right)-\tilde{y}_{h}(u), w_{h}\right), w_{h} \in W_{h}, \\
\left(\boldsymbol{p}_{h}\left(P_{h} u\right)-\boldsymbol{p}_{h}(u), \boldsymbol{v}_{h}\right)-\left(\tilde{y}_{h}\left(P_{h} u\right)-\tilde{y}_{h}(u), \operatorname{div} \boldsymbol{v}_{h}\right)=0, \boldsymbol{v}_{h} \in \boldsymbol{V}_{h}, \\
\quad\left(\operatorname{div}\left(\boldsymbol{p}_{h}\left(P_{h} u\right)-\boldsymbol{p}_{h}(u)\right), w_{h}\right)=\left(P_{h} u-u, w_{h}\right), w_{h} \in W_{h}, \\
\left(\tilde{\boldsymbol{q}}_{h}\left(P_{h} u\right)-\tilde{\boldsymbol{q}}_{h}(u), \boldsymbol{v}_{h}\right)-\left(z_{h}\left(P_{h} u\right)-z_{h}(u), \operatorname{div} \boldsymbol{v}_{h}\right)=0, \boldsymbol{v}_{h} \in \boldsymbol{V}_{h}, \\
\quad\left(\operatorname{div}\left(\tilde{\boldsymbol{q}}_{h}\left(P_{h} u\right)-\tilde{\boldsymbol{q}}_{h}(u)\right), w_{h}\right) \\
=\left(\tilde{z}_{h}\left(P_{h} u\right)-\tilde{z}_{h}(u), w_{h}\right)+\left(\tilde{y}_{h}\left(P_{h} u\right)-\tilde{y}_{h}(u), w_{h}\right), w_{h} \in W_{h}, \\
\quad\left(\boldsymbol{q}_{h}\left(P_{h} u\right)-\boldsymbol{q}_{h}(u), \boldsymbol{v}_{h}\right)-\left(\tilde{z}_{h}\left(P_{h} u\right)-\tilde{z}_{h}(u), \operatorname{div} \boldsymbol{v}_{h}\right) \\
=-\left(\tilde{\boldsymbol{p}}_{h}\left(P_{h} u\right)-\tilde{\boldsymbol{p}}_{h}(u), \boldsymbol{v}_{h}\right), \boldsymbol{v}_{h} \in \boldsymbol{V}_{h},
\end{gathered}
$$

Noticing that $\left(P_{h} u-u, w_{h}\right)=0$, then (3.45)-(3.48) follows from the standard stability estimate.

Now, we will discuss the superconvergence for the control variable.

Lemma 3.5. Let $u$ be the solution of (2.15)-(2.23) and $u_{h}$ be the solution of (2.40)-(2.48), respectively. Let all the assumptions of Lemma 3.2 be valid. Then, we have

$$
\left\|P_{h} u-u_{h}\right\| \leq C h^{k+2} .
$$


Proof. We choose $\tilde{u}=u_{h}$ in (2.23) and $\tilde{u}_{h}=P_{h} u$ in (2.48) to get the following two inequalities:

$$
\left(\nu u+z, u_{h}-u\right) \geq 0
$$

and

$$
\left(\nu u_{h}+z_{h}, P_{h} u-u_{h}\right) \geq 0 .
$$

Note that $u_{h}-u=u_{h}-P_{h} u+P_{h} u-u$. Adding the two inequalities (3.58) and (3.59), we have

$$
\left(\nu u_{h}+z_{h}-\nu u-z, P_{h} u-u_{h}\right)+\left(\nu u+z, P_{h} u-u\right) \geq 0 .
$$

Thus, by (3.60), we find that

$$
\begin{aligned}
\nu\left\|P_{h} u-u_{h}\right\|^{2} & =\nu\left(P_{h} u-u_{h}, P_{h} u-u_{h}\right) \\
& =\nu\left(P_{h} u-u, P_{h} u-u_{h}\right)+\nu\left(u-u_{h}, P_{h} u-u_{h}\right) \\
& \leq\left(z_{h}-z, P_{h} u-u_{h}\right)+\left(\nu u+z, P_{h} u-u\right) .
\end{aligned}
$$

Observe that

$\left(z_{h}-z, P_{h} u-u_{h}\right)=\left(z_{h}-z_{h}\left(P_{h} u\right), P_{h} u-u_{h}\right)+\left(z_{h}\left(P_{h} u\right)-z_{h}(u), P_{h} u-u_{h}\right)$

$$
+\left(z_{h}(u)-P_{h} z, P_{h} u-u_{h}\right) \text {. }
$$

By Lemma 3.2 and Lemma 3.4, we arrive at

$$
\left(z_{h}(u)-P_{h} z, P_{h} u-u_{h}\right) \leq C h^{2 k+4}+\frac{\nu}{4}\left\|P_{h} u-u_{h}\right\|^{2}
$$

and

$$
\left(z_{h}\left(P_{h} u\right)-z_{h}(u), P_{h} u-u_{h}\right)=0 .
$$

From (2.24), we know that

$$
\nu u+z=\max \{0, \bar{z}\}=\text { const. }
$$

Thus, we have

$$
\left(\nu u+z, P_{h} u-u\right)=(\nu u+z) \int_{\Omega}\left(P_{h} u-u\right)=0 .
$$

Combining (3.61)-(3.64), (3.66) with Lemma 3.3, we derive (3.57).

Now, we can derive the $L^{\infty}$-error estimates for the control variable.

Theorem 3.1. Let $u$ and $u_{h}$ be the solutions of (2.15)-(2.23) and (2.40)-(2.48). Let all the assumptions of Lemma 3.2 be valid. Assume that $u \in W^{k+1, \infty}(\Omega)$. Then we have

$$
\begin{aligned}
& \left\|u-u_{h}\right\| \leq C h^{k+1}, \\
& \left\|u-u_{h}\right\|_{0, \infty} \leq C h^{k+1} .
\end{aligned}
$$


ERROR ESTIMATES FOR FOURTH ORDER ELLIPTIC CONTROL PROBLEMS 1137

Proof. By (2.29), Lemma 3.5 and the inverse estimate, we have

$$
\left\|u-u_{h}\right\| \leq\left\|u-P_{h} u\right\|+\left\|P_{h} u-u_{h}\right\| \leq C h^{k+1}
$$

and

$$
\begin{aligned}
\left\|u-u_{h}\right\|_{0, \infty} & \leq\left\|u-P_{h} u\right\|_{0, \infty}+\left\|P_{h} u-u_{h}\right\|_{0, \infty} \\
& \leq C\left(h^{k+1}+h^{-1} \cdot\left\|P_{h} u-u_{h}\right\|\right) \leq C h^{k+1} .
\end{aligned}
$$

Theorem 3.2. Let $(\boldsymbol{p}, \tilde{y}, \tilde{\boldsymbol{p}}, y, \boldsymbol{q}, \tilde{z}, \tilde{\boldsymbol{q}}, z)$ and $\left(\boldsymbol{p}_{h}, \tilde{y}_{h}, \tilde{\boldsymbol{p}}_{h}, y_{h}, \boldsymbol{q}_{h}, \tilde{z}_{h}, \tilde{\boldsymbol{q}}_{h}, z_{h}\right)$ be the solutions of (2.15)-(2.23) and (2.40)-(2.48), respectively. Let all the assumptions of Lemma 3.2 be valid. Then we have

$$
\begin{aligned}
& \left\|y-y_{h}\right\|+\left\|\boldsymbol{p}-\boldsymbol{p}_{h}\right\|_{d i v} \leq C h^{k+1}, \\
& \left\|\tilde{y}-\tilde{y}_{h}\right\|+\left\|\tilde{\boldsymbol{p}}-\tilde{\boldsymbol{p}}_{h}\right\|_{\text {div }} \leq C h^{k+1}, \\
& \left\|z-z_{h}\right\|+\left\|\boldsymbol{q}-\boldsymbol{q}_{h}\right\|_{\text {div }} \leq C h^{k+1}, \\
& \left\|\tilde{z}-\tilde{z}_{h}\right\|+\left\|\tilde{\boldsymbol{q}}-\tilde{\boldsymbol{q}}_{h}\right\|_{\text {div }} \leq C h^{k+1} .
\end{aligned}
$$

Proof. First, we choose $\tilde{u}=u_{h}$ and $\tilde{u}=u$ in (2.49)-(2.56) respectively. We can obtain the following error equations

$$
\left(\tilde{\boldsymbol{p}}_{h}-\tilde{\boldsymbol{p}}_{h}(u), \boldsymbol{v}_{h}\right)-\left(y_{h}-y_{h}(u), \operatorname{div} \boldsymbol{v}_{h}\right)=0, \boldsymbol{v}_{h} \in \boldsymbol{V}_{h},
$$

$$
\left(\operatorname{div}\left(\tilde{\boldsymbol{p}}_{h}-\tilde{\boldsymbol{p}}_{h}(u)\right), w_{h}\right)=\left(\tilde{y}_{h}-\tilde{y}_{h}(u), w_{h}\right), w_{h} \in W_{h},
$$

$$
\left(\boldsymbol{p}_{h}-\boldsymbol{p}_{h}(u), \boldsymbol{v}_{h}\right)-\left(\tilde{y}_{h}-\tilde{y}_{h}(u), \operatorname{div} \boldsymbol{v}_{h}\right)=0, \boldsymbol{v}_{h} \in \boldsymbol{V}_{h},
$$

$$
\left(\operatorname{div}\left(\boldsymbol{p}_{h}-\boldsymbol{p}_{h}(u)\right), w_{h}\right)=\left(u_{h}-u, w_{h}\right), w_{h} \in W_{h},
$$

$$
\left(\tilde{\boldsymbol{q}}_{h}-\tilde{\boldsymbol{q}}_{h}(u), \boldsymbol{v}_{h}\right)-\left(z_{h}-z_{h}(u), \operatorname{div} \boldsymbol{v}_{h}\right)=0, \boldsymbol{v}_{h} \in \boldsymbol{V}_{h},
$$

$$
\left(\operatorname{div}\left(\tilde{\boldsymbol{q}}_{h}-\tilde{\boldsymbol{q}}_{h}(u)\right), w_{h}\right)=\left(\tilde{z}_{h}-\tilde{z}_{h}(u), w_{h}\right)+\left(\tilde{y}_{h}-\tilde{y}_{h}(u), w_{h}\right), w_{h} \in W_{h},
$$

$$
\left(\boldsymbol{q}_{h}-\boldsymbol{q}_{h}(u), \boldsymbol{v}_{h}\right)-\left(\tilde{z}_{h}-\tilde{z}_{h}(u), \operatorname{div} \boldsymbol{v}_{h}\right)=-\left(\tilde{\boldsymbol{p}}_{h}-\tilde{\boldsymbol{p}}_{h}(u), \boldsymbol{v}_{h}\right), \boldsymbol{v}_{h} \in \boldsymbol{V}_{h},
$$

$$
\left(\operatorname{div}\left(\boldsymbol{q}_{h}-\boldsymbol{q}_{h}(u)\right), w_{h}\right)=\left(y_{h}-y_{h}(u), w_{h}\right), w_{h} \in W_{h} .
$$

It follows from stability estimate that

$$
\begin{gathered}
\left\|\boldsymbol{p}_{h}-\boldsymbol{p}_{h}(u)\right\|_{\text {div }}+\left\|y_{h}-y_{h}(u)\right\| \leq C\left\|u-u_{h}\right\|, \\
\left\|\boldsymbol{q}_{h}-\boldsymbol{q}_{h}(u)\right\|_{\text {div }}+\left\|z_{h}-z_{h}(u)\right\| \leq C\left\|u-u_{h}\right\|, \\
\left\|\tilde{\boldsymbol{p}}_{h}-\tilde{\boldsymbol{p}}_{h}(u)\right\|_{\mathrm{div}}+\left\|\tilde{y}_{h}-\tilde{y}_{h}(u)\right\| \leq C\left\|u-u_{h}\right\|, \\
\left\|\tilde{\boldsymbol{q}}_{h}-\tilde{\boldsymbol{q}}_{h}(u)\right\|_{\mathrm{div}}+\left\|\tilde{z}_{h}-\tilde{z}_{h}(u)\right\| \leq C\left\|u-u_{h}\right\| .
\end{gathered}
$$


Then (3.71)-(3.74) follows from (3.83)-(3.86), Lemma 3.1 and Theorem 3.1.

Theorem 3.3. Let $(\boldsymbol{p}, \tilde{y}, \tilde{\boldsymbol{p}}, y, \boldsymbol{q}, \tilde{z}, \tilde{\boldsymbol{q}}, z)$ and $\left(\boldsymbol{p}_{h}, \tilde{y}_{h}, \tilde{\boldsymbol{p}}_{h}, y_{h}, \boldsymbol{q}_{h}, \tilde{z}_{h}, \tilde{\boldsymbol{q}}_{h}, z_{h}\right)$ be the solutions of (2.15)-(2.23) and (2.40)-(2.48), respectively. Let all the as-

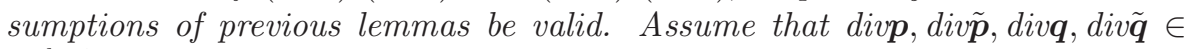
$W^{k+1, \infty}(\Omega)$. Then we have

$$
\begin{aligned}
&\left\|y-y_{h}\right\|_{0, \infty}+\left\|\operatorname{div}\left(\boldsymbol{p}-\boldsymbol{p}_{h}\right)\right\|_{0, \infty} \leq C h^{k+1}, \\
&\left\|\tilde{y}-\tilde{y}_{h}\right\|_{0, \infty}+\left\|\operatorname{div}\left(\tilde{\boldsymbol{p}}-\tilde{\boldsymbol{p}}_{h}\right)\right\|_{0, \infty} \leq C h^{k+1}, \\
&\left\|z-z_{h}\right\|_{0, \infty}+\left\|\operatorname{div}\left(\boldsymbol{q}-\boldsymbol{q}_{h}\right)\right\|_{0, \infty} \leq C h^{k+1}, \\
&\left\|\tilde{z}-\tilde{z}_{h}\right\|_{0, \infty}+\left\|\operatorname{div}\left(\tilde{\boldsymbol{q}}-\tilde{\boldsymbol{q}}_{h}\right)\right\|_{0, \infty} \leq C h^{k+1} .
\end{aligned}
$$

Proof. Using (2.31), we rewrite (3.19)-(3.26) as

$(3.96)\left(\operatorname{div}\left(\Pi_{h} \tilde{\boldsymbol{q}}-\tilde{\boldsymbol{q}}_{h}\right), w_{h}\right)=\left(P_{h} \tilde{z}-\tilde{z}_{h}, w_{h}\right)+\left(P_{h} \tilde{y}-\tilde{y}_{h}, w_{h}\right), \forall w_{h} \in W_{h}$,

$$
\begin{gathered}
\left(\tilde{\boldsymbol{p}}-\tilde{\boldsymbol{p}}_{h}, \boldsymbol{v}_{h}\right)-\left(P_{h} y-y_{h}, \operatorname{div} \boldsymbol{v}_{h}\right)=0, \forall \boldsymbol{v}_{h} \in \boldsymbol{V}_{h}, \\
\left(\operatorname{div}\left(\Pi_{h} \tilde{\boldsymbol{p}}-\tilde{\boldsymbol{p}}_{h}\right), w_{h}\right)=\left(P_{h} \tilde{y}-\tilde{y}_{h}, w_{h}\right), \forall w_{h} \in W_{h}, \\
\left(\boldsymbol{p}-\boldsymbol{p}_{h}, \boldsymbol{v}_{h}\right)-\left(P_{h} \tilde{y}-\tilde{y}_{h}, \operatorname{div} \boldsymbol{v}_{h}\right)=0, \forall \boldsymbol{v}_{h} \in \boldsymbol{V}_{h},
\end{gathered}
$$

Similar to Lemma 3.2, we can prove that

$$
\begin{aligned}
& \left\|P_{h} y-y_{h}\right\|+\left\|P_{h} z-z_{h}\right\| \leq C h^{k+2}, \\
& \left\|P_{h} \tilde{y}-\tilde{y}_{h}\right\|+\left\|P_{h} \tilde{z}-\tilde{z}_{h}\right\| \leq C h^{k+2} .
\end{aligned}
$$

By use of (2.29), (3.99)-(3.100) and the inverse estimate, we find that

$$
\begin{aligned}
& \left\|y-y_{h}\right\|_{0, \infty}+\left\|z-z_{h}\right\|_{0, \infty} \leq C h^{k+1}, \\
& \left\|\tilde{y}-\tilde{y}_{h}\right\|_{0, \infty}+\left\|\tilde{z}-\tilde{z}_{h}\right\|_{0, \infty} \leq C h^{k+1} .
\end{aligned}
$$

, (3.96) and (3.98), it is easy to derive that

$$
\begin{aligned}
& \operatorname{div}\left(\Pi_{h} \tilde{\boldsymbol{p}}-\tilde{\boldsymbol{p}}_{h}\right)=P_{h} \tilde{y}-\tilde{y}_{h}, \\
& \operatorname{div}\left(\Pi_{h} \boldsymbol{p}-\boldsymbol{p}_{h}\right)=P_{h} u-u_{h}, \\
& \operatorname{div}\left(\Pi_{h} \tilde{\boldsymbol{q}}-\tilde{\boldsymbol{q}}_{h}\right)=P_{h} \tilde{y}-\tilde{y}_{h}+P_{h} \tilde{z}-\tilde{z}_{h}, \\
& \operatorname{div}\left(\Pi_{h} \boldsymbol{q}-\boldsymbol{q}_{h}\right)=P_{h} y-y_{h} .
\end{aligned}
$$

Combining (2.33), (3.101)-(3.106) with the inverse estimate, we complete the proof of Theorem 3.3. 
Then, we introduce some notations. For any $x_{0} \in \Omega$, let

$$
\rho\left(x, x_{0}\right)=\left(\left|x-x_{0}\right|^{2}+|\theta|^{2}\right)^{\frac{1}{2}},
$$

where $x=\left(x_{1}, x_{2}\right) \in R^{2}, \theta=\gamma h$ and $\gamma$ is a positive number. The weighted function $\rho\left(\cdot, x_{0}\right)$ which was first introduced by Frehse and Rannacher [12], satisfies the following properties (see also [20]):

$$
\begin{gathered}
\max _{x \in T} \rho\left(x, x_{0}\right) \leq c \min _{x \in T} \rho\left(x, x_{0}\right), \quad \forall T \in T_{h}, \quad x_{0} \in \Omega, \\
\left|D^{j} \rho^{\alpha}\right| \leq c \rho^{\alpha-|j|}, \quad \alpha \text { is a real number, } \\
I_{\alpha}=\int_{\Omega} \rho^{\alpha} d x \leq \begin{cases}\frac{2}{\alpha+2}, & \alpha>-2 \\
c|\ln \theta|, & \alpha=-2, \\
\frac{c \theta^{2+\alpha}}{-\alpha-2}, & \alpha<-2 .\end{cases}
\end{gathered}
$$

The Green's function $\left\{\mathbf{G}_{2}\left(x, x_{0}\right), \lambda_{2}\left(x, x_{0}\right)\right\}$ is defined by

$$
\left\{\begin{aligned}
\mathbf{G}_{2}+\nabla \lambda_{2}=\boldsymbol{\delta}_{2}^{h} & \text { in } \Omega, \\
\operatorname{div} \mathbf{G}_{2}=0 & \text { in } \Omega, \\
\lambda_{2}=0 & \text { on } \partial \Omega,
\end{aligned}\right.
$$

where $\boldsymbol{\delta}_{2}^{h}=\boldsymbol{\delta}_{2}^{h}\left(x, x_{0}\right)$ is either $\left(\delta_{2}^{h}, 0\right)$ or $\left(0, \delta_{2}^{h}\right) ; \delta_{2}^{h}$ is a regularized Dirac $\delta$ function at $x_{0}$ satisfying

$$
\begin{aligned}
& \delta_{2}^{h}\left(\cdot, x_{0}\right) \in C^{1}(\Omega), \quad \operatorname{supp} \delta_{2}^{h} \subset E, \\
& \delta_{2}^{h} \geq 0, \quad \int_{\Omega} \delta_{2}^{h} d x=1, \\
& \left\|D^{j} \delta_{2}^{h}\right\|_{0, \infty} \leq c h^{-2-|j|}, \quad|j|=0,1 .
\end{aligned}
$$

Here, $E \subset T$ for some element $T, x_{0} \in E, E$ is required to have the following properties:

(i) $\operatorname{diam} E=A_{1} h_{T}$, with $A_{1}$ to be determined later,

(ii) there exists a ball $B$ with radius $A_{2} h_{T}$ such that $B \subset E$,

(iii) $E$ is star-shaped with respect to $B$.

The regularized Green's function $\left\{\mathbf{G}_{2}\left(x, x_{0}\right), \lambda_{2}\left(x, x_{0}\right)\right\}$ was first introduced by Wang $[11,21]$. $A_{1}\left(0<A_{1}<1\right)$ was chosen such that for $x_{0} \in T$ and $T \in T_{h}$ satisfying $[11,(3.11)]$

$$
\|\boldsymbol{v}\|_{0, \infty}=\max _{1 \leq i \leq 2}\left|v_{i}\right|_{0, \infty} \leq 2\left|\left(\boldsymbol{v}, \boldsymbol{\delta}_{2}^{h}\right)\right|, \quad \forall \boldsymbol{v} \in \boldsymbol{V}_{h} .
$$

Let $\left\{\mathbf{G}_{2}^{h}, \lambda_{2}^{h}\right\}$ be the mixed finite element approximation of $\left\{\mathbf{G}_{2}, \lambda_{2}\right\}$. Then the error equations are

$$
\left(\mathbf{G}_{2}-\mathbf{G}_{2}^{h}, \boldsymbol{v}_{h}\right)-\left(\lambda_{2}-\lambda_{2}^{h}, \operatorname{div} \boldsymbol{v}_{h}\right)=0, \forall \boldsymbol{v}_{h} \in \boldsymbol{V}_{h}
$$


and

$$
\left(\operatorname{div}\left(\mathbf{G}_{2}-\mathbf{G}_{2}^{h}\right), w_{h}\right)=0, \forall w_{h} \in W_{h} .
$$

From [4], we recall the following $L^{1}$-error estimates for the Green's functions and their mixed finite element approximation.

Lemma 3.6. For the second regularized Green's function $\left\{\mathbf{G}_{2}, \lambda_{2}\right\}$ defined in (3.111), the following $L^{1}$-estimate of boundness holds:

$$
\left\|\lambda_{2}\right\|_{0,1} \leq c .
$$

Lemma 3.7. Let $\left\{\mathbf{G}_{2}, \lambda_{2}\right\}$ and $\left\{\mathbf{G}_{2}^{h}, \lambda_{2}^{h}\right\}$ be as above. If the space index $k \geq 1$, then

$$
\left\|\mathbf{G}_{2}-\mathbf{G}_{2}^{h}\right\|_{0,1} \leq c
$$

Now, we can prove the following $L^{\infty}$-error estimates for the vector-valued functions.

Theorem 3.4. Let $\left(\boldsymbol{p}_{h}, \tilde{y}_{h}, \tilde{\boldsymbol{p}}_{h}, y_{h}, \boldsymbol{q}_{h}, \tilde{z}_{h}, \tilde{\boldsymbol{q}}_{h}, z_{h}\right) \in\left(\boldsymbol{V}_{h} \times W_{h}\right)^{4}$ be the solution of (2.49)-(2.56) and $(\boldsymbol{p}, \tilde{y}, \tilde{\boldsymbol{p}}, y, \boldsymbol{q}, \tilde{z}, \tilde{\boldsymbol{q}}, z) \in(\boldsymbol{V} \times W)^{4}$ be the solution of (2.15)-(2.23), respectively. Assume that $y, \tilde{y}, z, \tilde{z} \in W^{k+1, \infty}(\Omega), \boldsymbol{p}, \tilde{\boldsymbol{p}}, \boldsymbol{q}, \tilde{\boldsymbol{q}} \in$

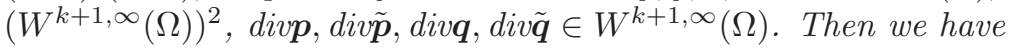

$$
\begin{aligned}
& \left\|\boldsymbol{p}-\boldsymbol{p}_{h}\right\|_{0, \infty}+\left\|\boldsymbol{q}-\boldsymbol{q}_{h}\right\|_{0, \infty} \leq C h^{k+1}, \\
& \left\|\tilde{\boldsymbol{p}}-\tilde{\boldsymbol{p}}_{h}\right\|_{0, \infty}+\left\|\tilde{\boldsymbol{q}}-\tilde{\boldsymbol{q}}_{h}\right\|_{0, \infty} \leq C h^{k+1} .
\end{aligned}
$$

Proof. Let

$$
\begin{aligned}
& \xi_{\boldsymbol{q}}=\Pi_{h} \boldsymbol{q}-\boldsymbol{q}_{h}, \quad \mathbf{r}_{\boldsymbol{q}}=\boldsymbol{q}-\Pi_{h} \boldsymbol{q}, \\
& \xi_{\boldsymbol{p}}=\tilde{\boldsymbol{p}}-\tilde{\boldsymbol{p}}_{h}, \quad \xi_{y}=y-y_{h} .
\end{aligned}
$$

Then the error equations (3.97) and (3.98) are reduced into the following form

$$
\begin{aligned}
\left(\xi_{\boldsymbol{q}}, \boldsymbol{v}_{h}\right)-\left(e, \operatorname{div} \boldsymbol{v}_{h}\right) & =-\left(\xi_{\boldsymbol{p}}, \boldsymbol{v}_{h}\right)-\left(\mathbf{r}_{\boldsymbol{q}}, \boldsymbol{v}_{h}\right), \quad \forall \boldsymbol{v}_{h} \in \boldsymbol{V}_{h}, \\
\left(\operatorname{div} \xi \boldsymbol{q}, w_{h}\right) & =\left(\xi_{y}, w_{h}\right), \quad \forall w_{h} \in W_{h} .
\end{aligned}
$$

Also let

$$
\left\|\xi_{\boldsymbol{q}}\right\|_{0, \infty} \leq 2\left|\left(\xi_{\boldsymbol{q}}, \boldsymbol{\delta}_{2}^{h}\right)\right| .
$$

Noting (3.111), (3.116)-(3.117) and (3.122)-(3.124), we estimate $\left(\xi_{\boldsymbol{q}}, \boldsymbol{\delta}_{2}^{h}\right)$ as follows:

$$
\begin{aligned}
\left(\xi_{\boldsymbol{q}}, \boldsymbol{\delta}_{2}^{h}\right) & =\left(\xi_{\boldsymbol{q}}, \mathbf{G}_{2}+\nabla \lambda_{2}\right) \\
& =\left(\xi_{\boldsymbol{q}}, \mathbf{G}_{2}-\mathbf{G}_{2}^{h}\right)+\left(\xi \boldsymbol{q}, \mathbf{G}_{2}^{h}\right)+\left(\xi \boldsymbol{q}, \nabla \lambda_{2}\right) \\
& =\left(\lambda_{2}-\lambda_{2}^{h}, \operatorname{div} \xi_{\boldsymbol{q}}\right)+\left(e, \operatorname{div} \mathbf{G}_{2}^{h}\right)-\left(r_{\boldsymbol{q}}, \mathbf{G}_{2}^{h}\right)-\left(\xi \boldsymbol{p}, \mathbf{G}_{2}^{h}\right)-\left(\lambda_{2}, \operatorname{div} \xi_{\boldsymbol{q}}\right) \\
& =\left(r_{\boldsymbol{q}}, \mathbf{G}_{2}-\mathbf{G}_{2}^{h}\right)+\left(r_{\boldsymbol{q}}, \nabla \lambda_{2}-\boldsymbol{\delta}_{2}^{h}\right)-\left(\xi_{\boldsymbol{p}}, \mathbf{G}_{2}^{h}\right)-\left(\xi_{y}, \lambda_{2}^{h}\right) \\
& =\left(r_{\boldsymbol{q}}, \mathbf{G}_{2}-\mathbf{G}_{2}^{h}\right)-\left(\operatorname{div} r_{\boldsymbol{q}}, \lambda_{2}-\lambda_{2}^{h}\right)-\left(r_{\boldsymbol{q}}, \boldsymbol{\delta}_{2}^{h}\right)-\left(\xi_{\boldsymbol{p}}, \mathbf{G}_{2}^{h}\right)-\left(\xi_{y}, \lambda_{2}^{h}\right) \\
& =\left(r_{\boldsymbol{q}}, \mathbf{G}_{2}-\mathbf{G}_{2}^{h}\right)-\left(\operatorname{div} r_{\boldsymbol{q}}, \lambda_{2}-\lambda_{2}^{h}\right)-\left(r_{\boldsymbol{q}}, \boldsymbol{\delta}_{2}^{h}\right)
\end{aligned}
$$


ERROR ESTIMATES FOR FOURTH ORDER ELLIPTIC CONTROL PROBLEMS 1141

$$
\begin{aligned}
& +\left(\xi_{\boldsymbol{p}}, \mathbf{G}_{2}-\mathbf{G}_{2}^{h}\right)+\left(\xi_{y}, \lambda_{2}-\lambda_{2}^{h}\right) \\
& -\left(\operatorname{div} \xi \boldsymbol{p}, \lambda_{2}\right)-\left(\xi_{\boldsymbol{p}}, \boldsymbol{\delta}_{2}^{h}\right)-\left(\xi_{y}, \lambda_{2}\right) \\
\leq & \left(\left\|r_{\boldsymbol{q}}\right\|_{0, \infty}+\left\|\xi_{\boldsymbol{p}}\right\|_{0, \infty}\right)\left(\left\|\mathbf{G}_{2}-\mathbf{G}_{2}^{h}\right\|_{0,1}+\left\|\boldsymbol{\delta}_{2}^{h}\right\|_{0,1}\right) \\
& +\left(\|\operatorname{div} r \boldsymbol{q}\|+\left\|\xi_{y}\right\|\right)\left\|\lambda_{2}-\lambda_{2}^{h}\right\|+\left(\left\|\operatorname{div} \xi_{\boldsymbol{p}}\right\|_{0, \infty}+\left\|\xi_{y}\right\|_{0, \infty}\right)\left\|\lambda_{2}\right\|_{0,1} .
\end{aligned}
$$

By using (2.29), (2.32)-(2.33), (3.118)-(3.119), (3.125), [21, (3.12b)] and Theorems 3.2-3.3, we derive

$$
\left\|\xi_{\boldsymbol{q}}\right\|_{0, \infty} \leq C h^{k+1}+C\left\|\Pi_{h} \tilde{\boldsymbol{p}}-\tilde{\boldsymbol{p}}_{h}\right\|_{0, \infty} .
$$

Similarly, we can prove

$$
\begin{gathered}
\left\|\Pi_{h} \tilde{\boldsymbol{p}}-\tilde{\boldsymbol{p}}_{h}\right\|_{0, \infty} \leq C h^{k+1}, \\
\left\|\Pi_{h} \boldsymbol{p}-\boldsymbol{p}_{h}\right\|_{0, \infty} \leq C h^{k+1}, \\
\left\|\Pi_{h} \tilde{\boldsymbol{q}}-\tilde{\boldsymbol{q}}_{h}\right\|_{0, \infty} \leq C h^{k+1}
\end{gathered}
$$

Thus, combining (2.32) with (3.126)-(3.129), we complete the proof.

\section{Numerical experiments}

In this section, we present below an example to illustrate the theoretical results. The optimization problems were solved numerically by projected gradient methods, with codes developed based on AFEPack [15]. The discretization was already described in Section 2: the control function $u$ was discretized by piecewise polynomials of order $k$, whereas the state $(y, \boldsymbol{p})$ and the co-state $(z, \boldsymbol{q})$ were approximated by the order $k$ Raviart-Thomas mixed finite element functions. In our example, we choose the domain $\Omega=[0,1] \times[0,1], \nu=1$ and $k=1$.

Example. We consider the following two-dimensional fourth order elliptic optimal control problem

$$
\min _{u \in U_{a d}}\left\{\frac{1}{2}\|\tilde{y}\|^{2}+\frac{1}{2}\|\tilde{\boldsymbol{p}}\|^{2}+\frac{1}{2}\left\|y-y_{d}\right\|^{2}+\frac{1}{2}\|u\|^{2}\right\}
$$

subject to the state equation

$$
\begin{aligned}
& \tilde{\boldsymbol{p}}=-\nabla y, \quad x \in \Omega, \\
& \operatorname{div} \tilde{\boldsymbol{p}}=\tilde{y}, \quad x \in \Omega, \\
& \boldsymbol{p}=-\nabla \tilde{y}, \quad x \in \Omega, \\
& \operatorname{div} \boldsymbol{p}=f+u, \quad x \in \Omega, \\
& y=\tilde{y}=0, \quad x \in \partial \Omega,
\end{aligned}
$$

where

$$
\begin{aligned}
& y=\sin \left(\pi x_{1}\right) \sin \left(\pi x_{2}\right), \\
& z=\sin \left(2 \pi x_{1}\right) \sin \left(2 \pi x_{2}\right),
\end{aligned}
$$


TABLE 1. The $L^{2}$ error of Example on a sequential uniform refined meshes.

\begin{tabular}{|c|c|c|c|c|c|}
\hline Resolution & $\left\|u-u_{h}\right\|$ & $\left\|y-y_{h}\right\|$ & $\left\|\tilde{y}-\tilde{y}_{h}\right\|$ & $\left\|\boldsymbol{p}-\boldsymbol{p}_{h}\right\|$ & $\left\|\tilde{\boldsymbol{p}}-\tilde{\boldsymbol{p}}_{h}\right\|$ \\
\hline $16 \times 16$ & $2.9118 \mathrm{e}-03$ & $7.2665 \mathrm{e}-04$ & $1.4537 \mathrm{e}-02$ & $4.0309 \mathrm{e}-02$ & $2.0161 \mathrm{e}-03$ \\
\hline $32 \times 32$ & $7.2802 \mathrm{e}-04$ & $1.8201 \mathrm{e}-04$ & $3.6359 \mathrm{e}-03$ & $1.0091 \mathrm{e}-02$ & $5.0488 \mathrm{e}-04$ \\
\hline $64 \times 64$ & $1.8185 \mathrm{e}-04$ & $4.5420 \mathrm{e}-05$ & $9.0847 \mathrm{e}-04$ & $2.5352 \mathrm{e}-03$ & $1.2677 \mathrm{e}-04$ \\
\hline $128 \times 128$ & $4.5872 \mathrm{e}-05$ & $1.1338 \mathrm{e}-05$ & $2.2677 \mathrm{e}-04$ & $6.3159 \mathrm{e}-04$ & $3.1659 \mathrm{e}-05$ \\
\hline
\end{tabular}

$$
\begin{aligned}
u & =\max \{0, \bar{z}\}-z, \\
\tilde{y} & =2 \pi^{2} y \\
\tilde{\boldsymbol{p}} & =-\left(\begin{array}{l}
\pi \cos \left(\pi x_{1}\right) \sin \left(\pi x_{2}\right) \\
\pi \sin \left(\pi x_{1}\right) \cos \left(\pi x_{2}\right)
\end{array}\right), \\
\boldsymbol{p} & =-\left(\begin{array}{l}
2 \pi^{3} \cos \left(\pi x_{1}\right) \sin \left(\pi x_{2}\right) \\
2 \pi^{3} \sin \left(\pi x_{1}\right) \cos \left(\pi x_{2}\right)
\end{array}\right), \\
f & =4 \pi^{4} y-u, \\
y_{d} & =y-64 \pi^{4} z+4 \pi^{4} y-2 \pi^{2} y .
\end{aligned}
$$

In the numerical implementation, we choose the solution $u$ which satisfies $\int_{\Omega} u d x=0$. In Tables 1 and 2 , the $L^{2}$ and $L^{\infty}$ errors obtained on a sequence of uniformly refined meshes are shown, respectively. In Figure 1, the profile of the numerical solution of $u$ on the $64 \times 64$ mesh grid is plotted. The theoretical results can be observed clearly from the data.

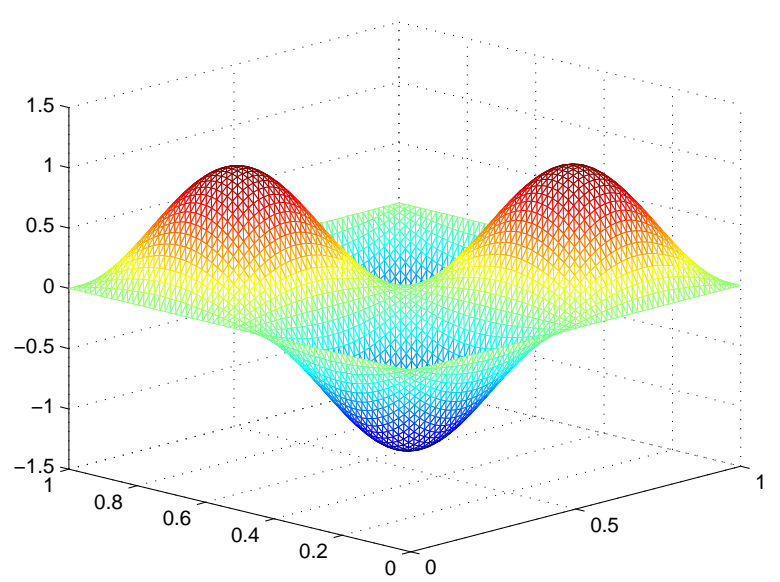

Figure 1. The profile of the numerical solution of Example on $64 \times 64$ triangle mesh. 
TABLE 2. The $L^{\infty}$ error of Example on a sequential uniform refined meshes.

\begin{tabular}{|c|c|c|c|c|c|}
\hline Resolution & $\left\|u-u_{h}\right\|_{0, \infty}$ & $\left\|y-y_{h}\right\|_{0, \infty}$ & $\left\|\tilde{y}-\tilde{y}_{h}\right\|_{0, \infty}$ & $\left\|\boldsymbol{p}-\boldsymbol{p}_{h}\right\|_{0, \infty}$ & $\left\|\tilde{\boldsymbol{p}}-\tilde{\boldsymbol{p}}_{h}\right\|_{0, \infty}$ \\
\hline $16 \times 16$ & $1.2812 \mathrm{e}-02$ & $3.1973 \mathrm{e}-03$ & $6.3966 \mathrm{e}-02$ & $1.7736 \mathrm{e}-01$ & $8.8710 \mathrm{e}-03$ \\
\hline $32 \times 32$ & $3.2033 \mathrm{e}-03$ & $7.9992 \mathrm{e}-04$ & $1.5998 \mathrm{e}-02$ & $4.4397 \mathrm{e}-02$ & $2.2215 \mathrm{e}-03$ \\
\hline $64 \times 64$ & $8.0017 \mathrm{e}-04$ & $1.9985 \mathrm{e}-04$ & $3.9973 \mathrm{e}-03$ & $1.1155 \mathrm{e}-02$ & $5.5778 \mathrm{e}-04$ \\
\hline $128 \times 128$ & $2.0184 \mathrm{e}-04$ & $4.9891 \mathrm{e}-05$ & $9.9782 \mathrm{e}-04$ & $2.7793 \mathrm{e}-03$ & $1.3930 \mathrm{e}-04$ \\
\hline
\end{tabular}

\section{References}

[1] F. Brezzi and M. Fortin, Mixed and Hybrid Finite Element Methods, Springer-Verlag, New York, 1991.

[2] H. Bium and R. Rannacher, On mixed finite element methods in plate bending analysis, Comput. Mech. 6 (1990), 221-236.

[3] W. Cao and D. Yang, Ciarlet-Raviart mixed finite element approximation for an optimal control problem governed by the first bi-harmonic equation, J. Comput. Appl. Math. 233 (2009), no. 2, 372-388.

[4] H. Chen and Z. Jiang, $L^{\infty}$-convergence of mixed finite element method for laplacian operator, Korean J. Comput. Appl. Math. 7 (2000), no. 1, 61-82.

[5] Y. Chen, Superconvergence of mixed finite element methods for optimal control problems, Math. Comp. 77 (2008), no. 263, 1269-1291.

[6] - Superconvergence of quadratic optimal control problems by triangular mixed finite elements, Internat. J. Numer. Methods Engrg. 75 (2008), no. 8, 881-898.

[7] Y. Chen, Y. Huang, W. B. Liu, and N. Yan, Error estimates and superconvergence of mixed finite element methods for convex optimal control problems, J. Sci. Comput. 42 (2009), no. 3, 382-403.

[8] Y. Chen and W. B. Liu, A posteriori error estimates for mixed finite element solutions of convex optimal control problems, J. Comput. Appl. Math. 211 (2008), no. 1, 76-89.

[9] X. L. Cheng, W. M. Han, and H. C. Huang, Some mixed finite element methods for biharmonic equation, J. Comput. Appl. Math. 126 (2000), no. 1-2, 91-109.

[10] J. Douglas and J. E. Roberts, Global estimates for mixed methods for second order elliptic equations, Math. Comp. 44 (1985), no. 169, 39-52.

[11] R. Duran, R. H. Nochetto, and J. Wang, Sharp maximum norm error estimates for FE approximations of the Stokes problems in 2-D, Math. Comp. 51 (1988), no. 184, 491-506.

[12] J. Frehse and R. Rannacher, Eine $L^{1}$-Ferhlerabschätzung für diskrete Grundlösungen in der Methode der Finiten Elemente, Finite Elemente (Tagung, Univ. Bonn, Bonn, 1975), pp. 92-114. Bonn. Math. Schrift., No. 89, Inst. Angew. Math., Univ. Bonn, Bonn, 1976.

[13] C. Johnson, On the convergence of a mixed finite-element method for plate bending problems, Numer. Math. 21 (1973), 43-62.

[14] B. J. Li and S. Y. Liu, On gradient-type optimization method utilizing mixed finite element approximation for optimal boundary control problem governed by bi-harmonic equation, Appl. Math. Comput. 186 (2007), no. 2, 1429-1440.

[15] R. Li and W. Liu, http://circus.math.pku.edu.cn/AFEPack.

[16] J. L. Lions, Optimal Control of Systems Governed by Partial Differential Equations, Springer-Verlag, Berlin, 1971.

[17] P. Monk, A mixed finite element method for the biharmonic equation, SIAM J. Numer. Anal. 24 (1987), no. 4, 737-749.

[18] P. A. Raviart and J. M. Thomas, A mixed finite element method for 2nd order elliptic problems, Mathematical aspects of finite element methods (Proc. Conf., Consiglio Naz. 
delle Ricerche (C.N.R.), Rome, 1975), pp. 292-315. Lecture Notes in Math., Vol. 606, Springer, Berlin, 1977.

[19] R. Scott, A Mixed method for 4th order problems using linear finite elements, RARIO Anal. Numer. 33 (1978), 681-697.

[20] Optimal $L^{\infty}$-estimates for the finite element method on irregular meshes, Math. Comp. 30 (1976), no. 136, 681-697.

[21] J. Wang, Asympotic expansion and $L^{\infty}$-error estimates for mixed FEM for second order elliptic problems, Numer. Math. 55 (1989), 401-430.

[22] X. Xing and Y. Chen, Error estimates of mixed finite element methods for quadratic optimal control problems, J. Comput. Appl. Math. 233 (2010), no. 8, 1812-1820.

School of Mathematical Sciences

South China Normal University

Guangzhou 510631, Guangdong, P. R. China

E-mail address: htlchb@163.com 